\title{
Impacto de la investigación y de los investigadores en comunicación en Latinoamérica: el índice $h$ de las revistas científicas
}

\section{Miguel Túñez-López' \\ Karina Valarezo-González ${ }^{2}$ \\ Isidro Marín-Gutiérrez ${ }^{3}$}

Recibido: 2014-03-12

Enviado a pares: 2014-03-28
Aprobado por pares: 2014-04-03

Aceptado: 2014-04-10

DOI: 10.5294/pacla.2014.17.3.14

Para citar este artículo / To reference this article / Para citar este artigo

Túñez-López, M., Valarezo-González, K., Marín-Gutiérrez, I. Septiembre 2014. Impacto de la investigación y de los investigadores en comunicación en Latinoamérica: el índice h de las revistas científicas. Palabra Clave 17 (3), 895-919. DOI: 10.5294/pacla.2014.17.3.14

\section{Resumen}

El índice $h$ es un indicador bibliométrico que señala el número h de publicaciones de un autor o una revista que han recibido un número h de citas. Este artículo revisa el factor de impacto con el índice h e indaga sobre la aplicación referencial al área de Comunicación y sobre el impacto h de las revistas científicas del área en Latinoamérica, España y Portugal..

\section{Palabras clave}

Índice H, Índice G, factor de impacto, Google ScholarMetrics, Scopus (Fuente: Tesauro de la Unesco).

\footnotetext{
Universidad de Santiago de Compostela USC, España. miguel.tunez@usc.es

Universidad Técnica Particular de Loja. Ecuador. kpvalarezo@utpl.edu.ec

Universidad de Huelva. España. isidro.marin@dstso.uhu.es
} 


\section{The Impact of Research and Researchers on Communication in Latin America: The $\mathrm{H}$-Index for Scientific Journals}

\section{Abstract}

The h-index is a bibliometric indicator that refers to the number of $\mathrm{h}$ citations an author or a journal has received in other publications. This article reviews the impact factor of the index, in addition to examining its application in the field of communication and the $h$ impact of scientific journals in Latin America, Spain and Portugal..

\section{Keywords}

H-index, g-index, impact factor, Google ScholarMetrics, Scopus (Source: UNESCO Thesaurus).

\section{Impacto da pesquisa e dos pesquisadores em Comunicação na América Latina: 0 índice h das revistas científicas}

\section{Resumo}

$\mathrm{O}$ índice h é um indicador bibliométrico que indica o número h de publicações de um autor ou uma revista que têm recebido um número h de citações. Este artigo revisa o fator de impacto com o índice h e questiona sobre a aplicação referencial à área de Comunicação e sobre o impacto h das revistas científicas da área na América Latina, Espanha e Portugal.

\section{Palavras-chave}

Índice H, Índice G, fator de impacto, Google Scholar Metrics, Scopus (Fonte: Tesauro da Unesco). 


\section{Introducción}

El índice $h$ es un indicador bibliométrico para medir la actividad investigadora y el impacto de su difusión, ya que equivale al dígito que equipara cuantitativamente las publicaciones de una revista o de un autor y las citas que éstas han obtenido. Propuesto en 2005 por el físico de la Universidad de California Jorge Hirsch, un investigador tiene índice $h$ si el valor de su número de artículos (NA) tiene al menos $h$ citas cada uno y los otros artículos (NA-h) tienen menos de $h$ citas cada uno (Hirsch, 2005). Es decir, hay un número $h$ de publicaciones de un autor que han recibido cada una de ellas, al menos, un número $h$ de citas. Un índice $h=20$, por ejemplo, quiere decir que hay un mínimo de 20 publicaciones que han recibido cada una de ellas al menos 20 citas.

El índice $h$ ha sido aceptado por la comunidad científica como una referencia bibliométrica válida porque, más allá de ser un marcador cuantitativo, combina la difusión con el impacto sobre el área, es fácil de calcular y resulta útil para identificar no sólo a las publicaciones sino también a los investigadores más destacados. Se trata de un indicador robusto que considera al mismo tiempo aspectos cuantitativos y cualitativos o de visibilidad (Dorta-González y Dorta-González, 2010, p. 226). No obstante, también se critica que no tenga en cuenta las citas que reciben los artículos con un número de referencias superiores a $h$, no diferencie entre investigadores emergentes y otros con una carrera detenida y que promocione a los productores masivos de artículos frente a los productores selectivos de pocos textos pero muy referenciados (Túñez, 2013).

Por eso, a partir de la propuesta de Hirsch han surgido otros indicadores complementarios para la correcta interpretación de $h$, entre ellos, el índice $h 5$, que refleja las citas recibidas en los últimos cinco años completos, independientemente del de publicación; el índice i10, que marca el número de artículos que han recibido al menos diez citas cada uno; el índice $g$ (Egghe, 2006), que compensa el impacto de los artículos con un número de citas superior al índice $h$; el índice $h /$ mediana, que refiere las citas del artículo central del $h$-core o grupo de publicaciones que determinan el $h$, y el índice 
$m$ (Hirsch, 2005), que divide $h$ entre el número de años de carrera investigadora para evitar que los investigadores noveles se vean desfavorecidos.

El índice $h$ ha tenido una rápida y favorable acogida entre la comunidad científica porque representa una alternativa sólida al factor de impacto. El factor de impacto es un indicador colectivo del soporte, no de los autores; concebido en 1963 por Eugene Garfield e Irving Sher como el Journal Impact Factor (JIF), mide la influencia de las revistas científicas a través del recuento del número de citas que han obtenido sus artículos en un periodo concreto de tiempo, inicialmente no determinado y posteriormente fijado por Garfield en dos años completos previos al año en que se realiza ese recuento. En cambio, el índice $h$ se asocia a la trayectoria de un investigador a través de las citas que reciben sus trabajos (Garfield, 2006).

Esta posibilidad de poder derivar el impacto desde la revista hacia el investigador sugiere que la visibilidad de la investigación, más allá de la publicación en soportes científicos, podría pasar a ser una responsabilidad compartida entre el editor de la revista y el/los autores de cada artículo, ya que las mediciones de impacto de ambos son independientes. De este modo comienza a hacerse patente la necesidad de comenzar a planificar estrategias de visibilidad que permitan abordar la publicación de resultados de investigación como una tarea de gestión integral de la comunicación, en lo que podría etiquetarse como un incipiente marketing de investigación 2.0 (Túñez, 2013, pp. 54-55).

Thomson Reuters, Elsevier y Google son las tres empresas multinacionales con las bases de datos más referenciadas para conocer el índice $h$. Las tres se basan en los resultados de motores de búsqueda en las revistas indexadas en su propia base de datos; es decir, usan universos de referencia diferentes para obtener sus indicadores, por lo que los índices $h$ de un mismo investigador o un mismo equipo pueden representarse con dígitos diferentes según el patrón de búsqueda que se utilice:

- La Web of Science (WoS) es la plataforma de la empresa Thomson Reuters, con productos analíticos y evaluativos (Journal Citations 


\section{Tabla 1 \\ Factor de impacto e índice $h$ en la evaluación de investigadores}

\begin{tabular}{|c|c|}
\hline Factor de impacto & Índice $\boldsymbol{h}$ \\
\hline $\begin{array}{c}\text { Es colectivo porque evalúa en conjunto a todos los } \\
\text { artículos incluidos en una misma revista. }\end{array}$ & $\begin{array}{c}\text { Es individual y colectivo: Investigadores, revistas, equipos } \\
\text { investigación... }\end{array}$ \\
\hline $\begin{array}{c}\text { Se refiere al soporte de difusión y se transfiere por igual a } \\
\text { todos los artículos, sin depender de una forma directa del } \\
\text { impacto individual de cada uno de ellos. }\end{array}$ & $\begin{array}{c}\text { Se refiere a las citas recibidas por cada texto y evalúa } \\
\text { el impacto de cada autor o equipo de acuerdo con los } \\
\text { impactos de los trabajos de cada uno de los actores } \\
\text { evaluados. }\end{array}$ \\
\hline $\begin{array}{c}\text { El impacto de un artículo está en función del impacto que } \\
\text { han logrado en grupo los artículos que se han publicado en } \\
\text { esa revista durante un período determinado. }\end{array}$ & $\begin{array}{c}\text { El impacto de un autor o de un equipo sólo depende del } \\
\text { impacto que ha conseguido con su difusión científica } \\
\text { propia, evaluada de manera individual o colectiva. }\end{array}$ \\
\hline $\begin{array}{c}\text { El autor se involucra en el proceso de difundir los } \\
\text { resultados hasta que su artículo es aceptado. }\end{array}$ & $\begin{array}{c}\text { Las 'gestión de la investigación' se amplía con la 'gestión de } \\
\text { la comunicación de la investigación difundida' porque lo } \\
\text { que revierte en el individuo o en el colectivo es el impacto } \\
\text { de lo difundido. }\end{array}$ \\
\hline $\begin{array}{c}\text { Las estrategias de visibilidad de los artículos con resultados } \\
\text { científicos corresponden a las revistas y el beneficio es } \\
\text { común para todos los autores que publican. }\end{array}$ & $\begin{array}{c}\text { La visibilidad del artículo es una responsabilidad que } \\
\text { comparten la revista y los autores porque cada cita computa } \\
\text { para el artículo y, también, para los indicadores del autor y } \\
\text { de la revista. }\end{array}$ \\
\hline
\end{tabular}

Fuente: Túñez, 2013, p. 64.

Report -JCR- o Essential Science Indicators) y de gestión de referencias bibliográficas (Endnote Web).

Scopus es la base de datos de Elsevier, lanzada en noviembre de 2004, formada por publicaciones seleccionadas por los 20 científicos y diez bibliotecarios de su Content Selection and Advisory Board (CSAB), y ofrece resultados de impacto con indicador SJR (SCImago Journal Rank), que pondera el valor de la cita según el prestigio (o valor SJR) que tiene la publicación en la que aparece.

- Google Scholar (GS) funciona desde 2004, pero empezó a ofrecer el servicio de Google Scholar Metrics (GSM) con datos de índice $h 5$ en abril de 2012, cuando presentó un primer ranking de revistas en alemán, chino, coreano, español, francés, holandés, inglés, italiano, japonés y portugués. En noviembre de 2012 se actualizó y corrigió 
errores de su primera oleada e incluyó un listado por áreas temáticas de revistas en inglés. Se renovaron ambos en julio de 2013.

La comparativa entre Google, Thomson Reuters y Elsevier que Delgado y Repiso (2013, p. 48) realizaron sobre la base del listado de Google de noviembre de 2012 deja clara esa diferencia. Ellos identificaron 664 revistas de comunicación en todo el mundo, de las que casi la mitad aparecen en la lista de GSM (Google Scholar Metrics) de noviembre 2012, pero en Scopus sólo están una de cada cuatro y en WoS no llegan a dos de cada diez. La supremacía anglosajona en WoS y Scopus queda visiblemente amortiguada en Google y la difusión en español casi se triplica en GSM sobre las otras dos bases. GSM indexa más revistas, de más países y en más idiomas y mide las revistas de forma muy parecida a los clásicos sistemas de evaluación de revistas (WoS y Scopus), por lo que, como sostienen Delgado y Repiso (2013, p. 51), en líneas generales, es una alternativa tan fiable y válida como éstos para medir el impacto de las revistas.

Tabla 2

Revistas de comunicación

\begin{tabular}{|c|c|c|c|c|}
\hline \multicolumn{2}{|c|}{$\begin{array}{l}\text { COMUNICACIÓN } \\
\text { (664 revistas) }\end{array}$} & $\begin{array}{c}\text { GSM } \\
(\text { Google })\end{array}$ & $\begin{array}{c}\text { Scopus } \\
\text { (Elsevier) }\end{array}$ & $\begin{array}{c}\text { WoS } \\
\text { (T. Reuters) }\end{array}$ \\
\hline \multicolumn{2}{|c|}{ Revistas } & $277(41,71 \%)$ & $167(25,15 \%)$ & $106(15,96 \%)$ \\
\hline \multicolumn{2}{|c|}{ Países } & 30 & 16 & 23 \\
\hline \multirow{4}{*}{ Idiomas } & Todos & 13 & 7 & 7 \\
\hline & Revistas en inglés & $59,67 \%$ & $88,66 \%$ & $86,92 \%$ \\
\hline & Revistas en español & $13,77 \%$ & $5,15 \%$ & $5,61 \%$ \\
\hline & Revistas en portugués & $7,78 \%$ & $1,55 \%$ & $0,00 \%$ \\
\hline
\end{tabular}

Fuente: elaboración propia a partir de Delgado y Repiso (2013).

Aunque los tres forman parte de las estrategias de mercado de tres multinacionales, Google universaliza el acceso, la búsqueda y la difusión al ser de uso gratuito, aunque su metodología es cuestionada por los expertos en bibliometría. La propuesta de GSM ha avivado el debate sobre la dependencia de la comunidad investigadora de los criterios no siempre claros de empresas privadas que tienen su modelo de negocio en la evaluación de la difusión de la actividad investigadora. Esa estrategia de mercado se basa en 
la utilidad del servicio que Thomson Reuters y Elsevier ofrecen casi a modo de monopolio internacional que se ve amenazado ahora por la estrategia de Google, basada en la audiencia del servicio. El eje de negocio se desplaza de la rentabilidad por coste a la gestión de relaciones como producto, ya que se ofrece gratuidad y acceso libre, pero los usuarios se convierten en audiencias apetecibles para clientes que necesiten ejecutar operaciones relacionales online para promover productos, ideas, personas, servicios u organizaciones (Túnez y De Pablos, 2012, p. 134).

GSM ha sido acogido con interés pero con reproches porque valida todas las referencias que detectan sus motores de rastreo sin diferenciar el soporte de citación y sin posibilidad de excluir las autocitas, lo que alimenta el debate entre los que defienden que eso lo aleja de ser considerado un referente fiable de la calidad de la investigación difundida y los que hacen la lectura a la inversa, argumentando que democratiza el impacto porque iguala la validez de todas las citas y rechaza que sólo las revistas sean soportes válidos como referencias de calidad, porque en ese caso, como explican Roncallo-Dow, Uribe-Jongbloed y Calderón-Reyes, "el qué se estudia cuando se habla de comunicación, y el para qué se estudia queda confinado a lo que esas publicaciones consideren relevante o actual" (2013, p. 183). También se cuestiona que se base en una categorización realizada por la empresa sin explicitar el criterio para determinar áreas y disciplinas y que no revele en cuantas áreas se ha incluido a una revista. El área de comunicación en GSM aparece en humanidades, literatura y artes.

Google permite a los investigadores crear perfiles personales o de grupos o revistas, lo que facilita tener sistematizada la producción científica, enlazada a los artículos u otros textos difundidos, tener control de las citas recibidas y mantener activado un enlace con los trabajos en los que están incluidas esas citas, además de ofrecer la evaluación de la trayectoria del autor calculando su $i$, su $i 10 \mathrm{y}$ su $i h 5$. Es una forma de universalizar el acceso a la producción científica como herramienta de fácil uso con contenidos sin coste, que fomenta la transparencia, ya que las trayectorias investigadoras se hacen visibles a través de los perfiles no sólo para la comunidad científica sino para toda la comunidad académica universitaria, los sectores profesionales implicados en cada campo de investigación y la sociedad en general. 
El perfil es una herramienta de visibilidad para difundir las publicaciones, mejorar la visibilidad y el impacto, gestionar y mantener actualizado el currículum, tener indicadores bibliométricos personales como el índice $h$, saber quién cita a quién, recibir alertas y seguir a otros investigadores (Delgado y Torres, 2013). Sin embargo, como han evidenciado Delgado, Robinson-García y Torres-Salinas (2012), el impacto en Google puede ser manipulado con la creación de citas o referencias artificiales que se alberguen en archivos alojados en directorios rastreados por los motores de Google.

El índice $h$ es un indicador personal pero, como ya señalaba Hirsch al proponerlo, si se puede aplicar a todos los miembros de una colectividad también debe poder aplicarse a la comunidad en su conjunto. Es posible hablar de regencia de impacto $h$ para equipos de investigación y para revistas, aunque, como ya se indicó, las comparativas no son válidas entre colectivos de áreas diferentes (Hirsch, 2012).

Como indicador colectivo, $h$ tiene aplicaciones que quedan de manifiesto en el análisis del proceso de creación del perfil de la Revista Latina de Comunicación Social en GSM, que permite advertir algunos inconvenientes a tener en cuenta, también, en la creación de perfiles individuales (Túñez y De Pablos, 2013):

- Casi un 35\% de los artículos están ausentes en la búsqueda inicial con el nombre como cadena de palabras. Más de la mitad de los artículos aparecen duplicados.

- Los resultados aparecen como figuran en la base rastreada, lo que provoca que en una de cada cuatro entradas la referencia nominal aparezca alterada.

- $\quad$ Es muy frecuente que los caracteres especiales (comillas o cursivas, por ejemplo), sobre todo en los títulos, necesiten ser editados.

Se estima que la elaboración de un perfil colectivo y su publicación abierta en un buscador universal permite (Túñez y De Pablos, 2013): 
- Ordenar el impacto de los artículos y conocer los autores más referenciados.

- Identificar duplicidades de contenidos con otras publicaciones e identificar autores que han publicado el mismo texto en más de una revista.

- Corregir los errores de atribución de citas a un mismo artículo identificado por los buscadores de modo fragmentado enlazando los enlaces de ambos.

- Enlazar a la revista y a los autores que publican en ella y que tienen perfil.

- Multiplicar las posibilidades de cita, ya que el perfil es un directorio que se puede ordenar por título, por año o por citas y dispone de url propia.

- $\quad$ Aumentar la visibilidad de los contenidos porque el perfil es de acceso libre y universal, lo que democratiza la difusión de los resultados de investigación.

- Motivar la difusión en libre acceso como contribución social desvinculada de intereses arancelarios y promover la responsabilidad social y el compromiso ético de la publicación al gestionar información sobre sí mismas y su impacto.

\section{Metodología}

Se ha desarrollado el cálculo del índice referencial para el área y las publicaciones de comunicación y se lo ha comparado con indicadores de Google Scholar Metrics y del listado internacional de revistas de comunicación actualizado a finales de 2013 por EC3, el grupo de investigación que calcula en España IN-RECS, los índices de impacto de revistas de ciencias sociales aceptados por el Ministerio de Educación. La base de datos IN-RECS se basa en la indexación sistemática de las referencias bibliográficas citadas en los artículos publicados en 154 revistas españolas de antropología, biblioteconomía y documentación, ciencia política y de la administración, comunicación, economía, educación, geografía, psicología, sociología y ur- 
banismo. En el caso de comunicación se usan siete revistas fuente. ${ }^{4}$ También lo hemos comparado con Qualis, listado oficial del Ministerio de Educación de Brasil, para tener resultados diferenciados en Iberoamérica por publicaciones en español y en portugués. En este estudio se realizaron búsquedas Ulrich's International Directory, GSM, Communication \& Mass Media Complete, WoS y Scopus, usando como palabras clave 'comunicación' (teoría, historia, investigación), 'medios de comunicación' (prensa, radio, televisión), 'periodismo', 'medios audiovisuales', 'cine', 'discurso y mensaje periodístico,' 'publicidad y relaciones públicas'. Tras depurarlas manualmente, la información se actualizó en una hoja de datos de Excel, donde se procedió a la unificación de títulos y la eliminación de duplicados. También se compararon los resultados de GSM y EC3 y se verificaron los títulos seleccionados catalogados Qualis como revistas de ciencias sociales.

\section{Resultados}

\section{Índice $h$ del área de comunicación en España}

El índice $h$ es un indicador no válido para establecer comparaciones entre diferentes áreas, aunque es posible determinar un $h$ referencial $\left(h_{R}\right)$ para cada una de ellas, que siempre estará fuertemente relacionado con el factor de impacto de sus principales revistas. Imperial y Rodríguez-Navarro (2005, apéndice 1) parten de que, aunque el propio Hirsch sostiene que no hay un coeficiente normalizador entre áreas, del estudio matemático que fundamenta su propuesta de índice $h$ con datos para biología y física se puede interpretar que "podría haber una relación sencilla entre los $h$ máximos que se dan en estas áreas y los factores de impacto de las revistas características para estos campos" (; por eso explican que tras computar el índice $h$ de científicos altamente citados dentro de varios campos y los factores de impacto de las revistas que caracterizan el área se puede establecer una constante, que expresan en su fórmula de referencia: $h_{R}=16+11$ xIF $(2005$, p. $6)$. En sus explicaciones sobre la fórmula, también advierten que este sistema no es aplicable, salvo alguna excepción, a las ciencias sociales y a las humanidades, porque estas áreas carecen de bases de datos de revistas su-

4 http://ec3.ugr.es/in-recs/informacion/como1.htm [fecha de consulta: 4 de abril de 2014]. 
ficientemente extensas y por el gran peso que en estas áreas tiene la publicación de libros.

Los datos de IN-RECS 2011 (los últimos disponibles a principios de 2014) ofrecen IF de 23 revistas (tres de ellas con valor cero). Sin descuidar las advertencias sobre su utilidad en ciencias sociales, si aplicamos la fórmula de Imperial y Rodríguez-Navarro, usando como referencia la media de IF de todas las revistas de comunicación, obtendríamos un $h$ referencial de 17; si sólo tomamos en cuenta las revistas del primer cuartil, el $h$ de comunicación sería de 18. En ambos casos, representa un índice $h$ superior al de la mayoría de los investigadores y de las revistas científicas del área.

\section{Tabla 3 \\ Simulacro de 'índice $\boldsymbol{h}_{\mathrm{R}}$ ' del área de comunicación en España}

\begin{tabular}{|c|c|c|}
\hline \multicolumn{2}{|c|}{ FI } & $\mathrm{hR}(16+11 \mathrm{xIF})$ \\
\hline Medio, todas las revistas & 0,1033 & 17,14 \\
\hline Primer cuartil & 0,28 & 18,10 \\
\hline
\end{tabular}

Fuente: Elaboración propia.

La cifra podría considerarse como estimación de los valores referenciales en una supuesta 'normalización' de índice a efectos de comparación con otras áreas, teniendo en cuenta que, dentro de las ciencias sociales, se estima que el índice $h$ de economía es, por ejemplo, de 50. Como reconocen los expertos en bibliometría, en general los índices en ciencias sociales son muy inferiores a los de otras ciencias (Dorta-González, 2010). En otras ciencias, como física, está en torno a 100, y en biología y biomedicina se estima un referencial $h$ de 150 (Dorta-González, 2010, p. 231).

Apenas se dispone de datos del índice $h$ personal de los investigadores en comunicación en España. En general, el interés del área de comunicación por $h$ como referencia bibliométrica es muy reciente, teniendo en cuenta que el primer debate sobre índice $h$ en foros científicos específicos del área tuvo lugar a principios del mes de mayo de 2013 durante el II Congreso de la Sección de Métodos de Investigación de la Asociación Española de Investigación en Comunicación (AE-IC), que se celebró en Segovia. 
La primera referencia al $h$ personal de investigadores de comunicación se desprende de una investigación sobre los perfiles de investigadores de comunicación en GSM realizado sobre una muestra de socios de la Asociación Española de Investigación de la Comunicación (AE-IC).

\section{Ih de las revistas de comunicación en Iberoamérica}

Las revistas científicas de comunicación se aproximan a ese $h$ referencial para el área, aunque incluso las que tienen un $h$ más elevado se quedan un poco por debajo. Cada base de datos ofrece sus propios datos de $h$. Aquí manejamos las listas de GSM y los trabajos del grupo EC3 sobre revistas de comunicación porque son los que incluyen un espectro de publicaciones más amplio.

El listado de índice $h 5$ que ofrece GSM es de revistas que en cinco años han publicado al menos cien artículos y han tenido por lo menos una cita, aunque no aclara las bases a las que acude para otorgar las citas a los artículos. Está disponible por áreas para publicaciones en inglés. Para revistas que se publican en español se ofrece una lista única de cien títulos. De momento, GSM ha ofrecido listas propias en noviembre de 2012, en julio de 2013 y en junio de $2014 .{ }^{5}$ De estos últimos, en español, ${ }^{6}$ entre las cien revistas del listado de GSM sólo hay cuatro vinculadas a comunicación, aunque la primera es de documentación y comunicación y la segunda de educación y comunicación. Ninguna de ellas se edita en Latinoamérica.

Para las revistas en portugués, entre las cien primeras hay tres referencias que podrían vincularse con comunicación ${ }^{7}$ y que representan las únicas revistas latinoamericanas que se pueden encontrar en los listados de Google, ya que la presencia, como se destacó en castellano, se limita a publicaciones españolas. En portugués, Google no incorpora ninguna revista de Portugal.

5 Durante el proceso de edición del artículo, GSM publicó sus datos de 2014

$6 \mathrm{http}: / /$ scholar.google.es/citations?view_op=top_venues\&hl=es\&vq=es [fecha de consulta: 16 de diciembre de 2013].

7 http://scholar.google.es/citations?view_op=top_venues\&hl=es\&vq=pt [fecha de consulta: 16 de diciembre de 2013] 


\section{Tabla 4}

\section{Comunicación en las cien revistas en español con mayor índice $\boldsymbol{h 5}$, según GSM}

\begin{tabular}{|c|c|c|c|c|c|c|}
\hline \multicolumn{2}{|c|}{ Puesto } & \multirow{2}{*}{ Revista } & \multicolumn{2}{c|}{$\mathbf{2 0 1 4}$} & \multicolumn{2}{c|}{2013} \\
\cline { 1 - 5 } $\mathbf{2 0 1 4}$ & $\mathbf{2 0 1 3}$ & & h.5 & Mediana & h.5 & Mediana \\
\hline 4 & 40 & Comunicar & 19 & 29 & 13 & 16 \\
\hline 9 & 15 & El Profesional de la Información & 18 & 29 & 16 & 21 \\
\hline 25 & 43 & Revista Latina de Comunicación Social & 15 & 21 & 12 & 19 \\
\hline 99 & 70 & Telos & 10 & 13 & 11 & 14 \\
\hline 1 & & Psicothema & 27 & 37 & & 38 \\
\hline
\end{tabular}

Fuente: Google Scholar, 2013. Elaboración propia.

\section{Tabla 5 \\ Comunicación en las cien revistas en portugués con mayor índice $h 5$, según GSM}

\begin{tabular}{|c|c|c|c|c|c|c|}
\hline \multicolumn{2}{|c|}{ Puesto } & \multicolumn{2}{c|}{2014} & \multicolumn{2}{c|}{2013} \\
\cline { 1 - 5 } $\mathbf{2 0 1 4}$ & $\mathbf{2 0 1 3}$ & Revista & h.5 & Mediana & h.5 & Mediana \\
\hline 12 & 14 & Interface - Comunicaçao, Saúde, Educaçao & 23 & 28 & 21 & 26 \\
\hline 64 & 55 & El Profesional de la Información & 13 & 21 & 14 & 18 \\
\hline 82 & 77 & Revista Latina de Comunicación Social & 12 & 16 & 12 & 16 \\
\hline 1 & 1 & Cadernos de Saúde Pública & 39 & 49 & 38 & 50 \\
\hline
\end{tabular}

Fuente: Google Scholar, 2003. Elaboración propia.

El grupo EC3 publicó en noviembre de 2012 un listado mundial del índice $h 5$ de revistas de comunicación (Delgado y Repiso, 2012) elaborado con los listados y los datos de GSM, que actualizó en octubre de 2013 (Repiso y Delgado López-Cózar, 2013).

El número de publicaciones ascendió de 223 en 2012 a 354 en 2013, lo que significa un incremento del $58,7 \%$, con incorporaciones de nuevos títulos de España, Brasil, Portugal, Bolivia, Chile, Colombia y México. Es significativo que mientras en 2012, en el primer cuartil, sólo hay una revista de Iberoamérica, la brasileña Interface - Comunicação, Saúde, Educação, en 2013 ya se incorporan también Comunicar y Revista Latina de Comuni- 
cación Social a un primer cuartil dominado por revistas anglófonas, mayoritariamente de EE. UU., el Reino Unido y Canadá.

Aunque EC3 la incluye en sus listas, Interface - Comunicação, Saúde, Educação no aparece referenciada como tal en Qualis, el listado oficial de la Fundación CAPES (Coordenaçao de Aperfeiçoamento de Pessoal de Nivel Superior) del MEC (Ministerio da Educaçao), en el que se categoriza a las revistas en Brasil. El ISSN con el que se representa Interface en la Web, con el subtítulo de Comunicação, Saúde, Educação (1807-5762), ${ }^{8}$ conduce en el buscador de Qualis a 13 publicaciones de diferentes áreas de evaluación, entre las que no están comunicación ni ciencias sociales, por lo que realmente de comunicación sólo podría hablarse de dos revistas de Iberoamérica en ese primer cuartil: Comunicar y Revista Latina de Comunicación Social (tabla 6).

\section{Tabla 6 \\ Revistas iberoamericanas en el primer cuartil de la lista mundial de revistas de comunicación, según EC3}

\begin{tabular}{|c|c|c|c|c|c|c|}
\hline \multirow{2}{*}{ C } & País & $\begin{array}{c}\text { Puesto } \\
\mathbf{2 0 1 3} \\
(\mathbf{2 0 1 2})\end{array}$ & Revista & $\begin{array}{c}\text { h.5 } \\
\mathbf{2 0 0 8 /} \\
\mathbf{2 0 1 2}\end{array}$ & $\begin{array}{c}\text { Med. } \\
\text { ih.5 }\end{array}$ & $\begin{array}{c}\text { h.5 } \\
\mathbf{2 0 0 7 /} \\
\mathbf{2 0 1 1}\end{array}$ \\
\hline \multirow{3}{*}{1} & $\mathrm{Br}$ & $31(36)$ & Interface: Communication, Healt, Education & 21 & 26 & 19 \\
\cline { 2 - 8 } & $\mathrm{Es}$ & $80(93)$ & Comunicar & 13 & 16 & 9 \\
\cline { 2 - 8 } & $\mathrm{Es}$ & $84(90)$ & Revista Latina de Comunicación Social & 12 & 19 & 9 \\
\hline
\end{tabular}

Fuente: Repiso y Delgado, 2013; Delgado y Cabezas-Clavijo, 2012. Elaboración propia.

El segundo cuartil está dominado por Brasil (siete revistas) y España (seis revistas). Sobre el listado de 2012, en 2013 se incorpora al segundo cuartil una publicación de Portugal, Observatorio, otra de Colombia, Palabra Clave, y otra más de México, Razón y Palabra. Ninguna de las tres figuraba en la lista anterior. Aparece también Comunicación y Sociedad, de la Universidad de Navarra, y la brasileña MATRIZes.

8 http://www.interface.org.br. 


\section{Tabla 7}

\section{Revistas iberoamericanas en el segundo cuartil de la lista de mundial de revistas de comunicación, según EC3}

\begin{tabular}{|c|c|c|c|c|c|c|}
\hline C & País & $\begin{array}{c}\text { Puesto } \\
2013 \\
(2012)\end{array}$ & Revista & $\begin{array}{c}h .5 \\
2008 / \\
2012\end{array}$ & $\begin{array}{c}\text { Med. } \\
\text { ih.5 }\end{array}$ & $\begin{array}{c}h .5 \\
2007 / \\
2011\end{array}$ \\
\hline \multirow{15}{*}{2} & $\mathrm{Br}$ & $-(75)$ & Perspectivas em Ciência da Informação & $(-)$ & $(-)$ & 11 \\
\hline & Es & $98(86)$ & Telos: Cuadernos de comunicación e innovación & 11 & 14 & 10 \\
\hline & $\mathrm{Br}$ & $122(-)$ & MATRIZes & 9 & 14 & $(-)$ \\
\hline & $\mathrm{Br}$ & $122(-)$ & Revista FAMECOS: mídia, cultura e tecnologia & 9 & 14 & $(-)$ \\
\hline & Es & $141(117)$ & Estudios sobre el Mensaje Periodístico & 8 & 10 & 7 \\
\hline & Po & $141(-)$ & Observatorio & 8 & 10 & $(-)$ \\
\hline & $\mathrm{Br}$ & $145(91)$ & Comunicação, Mídia e Consumo & 8 & 9 & 9 \\
\hline & $\mathrm{Br}$ & $149(99)$ & Comunicação \& Educação & 7 & 11 & 8 \\
\hline & $\mathrm{Br}$ & $149(130)$ & Intercom-Revista Brasileira de Ciências da Comunicação & 7 & 11 & 6 \\
\hline & $\mathrm{Br}$ & $152(101)$ & Comunicação em ciências da saúde & 7 & 10 & 8 \\
\hline & Es & $152(111)$ & $\begin{array}{l}\text { Zer: Revista de estudios de comunicación = } \\
\text { Komunikazio ikasketen aldizkaria }\end{array}$ & 7 & 10 & 7 \\
\hline & Es & $157(-)$ & Comunicación y Sociedad & 7 & 9 & $(-)$ \\
\hline & $\mathrm{Mx}$ & $157(-)$ & Razón y Palabra & 7 & 9 & $(-)$ \\
\hline & $\mathrm{Br}$ & $170(-)$ & Informação \& Sociedade & 7 & 8 & $(-)$ \\
\hline & Col & $175(-)$ & Palabra Clave & 7 & 7 & $(-)$ \\
\hline
\end{tabular}

Fuente: Repiso y Delgado, 2013; Delgado y Cabezas-Clavijo, 2012. Elaboración propia.

El tercer cuartil también es mayoritariamente brasileño. Son significativas las apariciones de nuevos títulos de revistas colombianas (Signo y Pensamiento), venezolanas (Opción y REDHECS) y una de Chile (Cuadernos de Información) - país del que no había referenciada ninguna en el listado de 2012-, y la correcta adscripción a Perú de Diálogos de la Comunicación, que en el listado anterior aparecía como española y peruana. Como se indicó, debe considerarse anecdótica la variación de posición en la tabla porque el número global de publicaciones aumenta considerablemente.

En el último cuartil hay publicaciones de varios países, entre las que destaca la inclusión de una revista de Bolivia (Punto Cero), una segunda chilena (F@ro) y una mexicana (Cineforum), porque aumentan la presencia de títulos latinoamericanos en la referencia de revistas científicas. También está en este cuartil la única revista de Ecuador (Chasqui - Revista Latinoa- 


\section{Tabla 8}

\section{Revistas iberoamericanas en el tercer cuartil de la lista mundial de revistas de comunicación, según EC3}

\begin{tabular}{|c|c|c|c|c|c|c|}
\hline C & País & $\begin{array}{c}\text { Puesto } \\
2013 \\
(2012)\end{array}$ & Revista & $\begin{array}{c}\text { h.5 } \\
2008 / \\
2012 \\
\end{array}$ & $\begin{array}{r}\text { Med. } \\
\text { ih.5 }\end{array}$ & $\begin{array}{r}\text { h.5 } \\
2007 / \\
2011\end{array}$ \\
\hline \multirow{26}{*}{3} & $\mathrm{Br}$ & $184(-)$ & Líbero & 6 & 9 & $(-)$ \\
\hline & $\mathrm{Col}$ & $184(-)$ & Signo y Pensamiento & 6 & 9 & $(-)$ \\
\hline & $\mathrm{Br}$ & $-(124)$ & $\begin{array}{l}\text { Economia Política das Tecnologias da Informação e da } \\
\text { Comunicação }\end{array}$ & $(-)$ & $(-)$ & 6 \\
\hline & Mex & $193(130)$ & Comunicación y Sociedad & 6 & 7 & 6 \\
\hline & Es & $193(130)$ & Trípodos & 6 & 7 & 6 \\
\hline & $\mathrm{Br}$ & 207 (139) & Estudos em Jornalismo e Mídia & 5 & 9 & 5 \\
\hline & $\mathrm{Br}$ & $209(-)$ & Revista Organicom & 5 & 9 & $(-)$ \\
\hline & $\mathrm{Br}$ & $211(137)$ & Brazilian Journalism Research & 5 & 8 & 5 \\
\hline & Es & $211(143)$ & CIC: Cuadernos de información y comunicación & 5 & 8 & 5 \\
\hline & $\mathrm{Br}$ & $211(-)$ & Eptic & 5 & 8 & $(-)$ \\
\hline & Es & $211(179)$ & Revista ICONO14 & 5 & 8 & 3 \\
\hline & Es & $216(-)$ & Doxa Comunicación & 5 & 7 & $(-)$ \\
\hline & Es & $216(155)$ & $\begin{array}{l}\text { Pensar la publicidad: revista internacional de } \\
\text { investigaciones publicitarias }\end{array}$ & 5 & 7 & 4 \\
\hline & $\mathrm{Br}$ & $226(161)$ & Comunicação \& Sociedade & 5 & 6 & 4 \\
\hline & $\mathrm{Br}$ & $226(161)$ & Contemporanea-Revista de Comunicação e Cultura & 5 & 6 & 4 \\
\hline & $\mathrm{Pe}$ & $241(143)$ & Diálogos de la Comunicación & 4 & 9 & 5 \\
\hline & Ven & $241(-)$ & Opción & 4 & 9 & $(-)$ \\
\hline & Ven & $244(-)$ & REDHECS & 4 & 8 & $(-)$ \\
\hline & Es & $246(177)$ & Comunicación y Hombre & 4 & 7 & 3 \\
\hline & Es & $251(158)$ & Ámbitos: Revista internacional de Comunicación & 4 & 6 & 4 \\
\hline & Chi & $251(-)$ & Cuadernos de Información & 4 & 6 & $(-)$ \\
\hline & $\mathrm{Br}$ & $251(-)$ & Intexto & 4 & 6 & $(-)$ \\
\hline & $\mathrm{Br}$ & $251(-)$ & Revista Contracampo & 4 & 6 & $(-)$ \\
\hline & $\mathrm{Br}$ & $260(171)$ & Conexão & 4 & 5 & 4 \\
\hline & $\mathrm{Br}$ & $260(135)$ & Distúrbios da Comunicação & 4 & 5 & 5 \\
\hline & $\mathrm{Br}$ & $260(-)$ & Em Questão & 4 & 5 & $(-)$ \\
\hline
\end{tabular}

Fuente: Repiso y Delgado, 2013; Delgado y Cabezas-Clavijo, 2012. Elaboración propia.

mericana de Comunicación). Aunque la Universidad de Medellín también edita una revista, Anagramas, en el listado se identifica como colombiana a Anagrama: Revista Científica Interdisciplinar da Graduação, que con ese tí- 
tulo publica el Departamento de Jornalismo e Editoração de la Escuela de Comunicaciones y Artes de la Universidad de São Paulo.

\section{Tabla 9}

\section{Revistas iberoamericanas en el último cuartil de la lista mundial de revistas de comunicación, según EC3}

\begin{tabular}{|c|c|c|c|c|c|c|}
\hline C & País & $\begin{array}{l}\text { Puesto } \\
\text { actual } \\
\text { (antes) }\end{array}$ & Revista & $\begin{array}{c}h .5 \\
2008 / \\
2012\end{array}$ & $\begin{array}{l}\text { Med. } \\
\text { ih.5 }\end{array}$ & $\begin{array}{c}\text { h.5 } \\
2007 / \\
2011\end{array}$ \\
\hline \multirow{26}{*}{4} & Es & $271(137)$ & Anàlisi: quaderns de comunicació i cultura & 4 & 4 & 5 \\
\hline & Es & $271(119)$ & $\begin{array}{c}\text { Comunicación y Pedagogía: Nuevas tecnologías y } \\
\text { recursos didácticos }\end{array}$ & 4 & 4 & 6 \\
\hline & $\mathrm{Es} / \mathrm{Ve}$ & $281(192)$ & $\begin{array}{l}\text { Anuario electrónico de estudios en Comunicación } \\
\text { Social. "Disertaciones" }\end{array}$ & 3 & 6 & 2 \\
\hline & Po & $281(176)$ & $\begin{array}{c}\text { DOC On-line: Revista Digital de Cinema } \\
\text { Documentário }\end{array}$ & 3 & 6 & 3 \\
\hline & $\mathrm{Mx}$ & $285(-)$ & Cineforum & 3 & 5 & $(-)$ \\
\hline & $\mathrm{Br}$ & $285(-)$ & Contemporânea & 3 & 5 & $(-)$ \\
\hline & $\mathrm{Br}$ & $285(-)$ & Discursos Fotográficos & 3 & 5 & $(-)$ \\
\hline & Ven & $292(182)$ & Comunicación: estudios venezolanos de comunicación & 3 & 4 & 3 \\
\hline & Es & $292(178)$ & Mediaciones & 3 & 4 & 3 \\
\hline & Es & $292(-)$ & Mediaciones Sociales Revista Electrónica & 3 & 4 & $(-)$ \\
\hline & Es & $292(-)$ & $\begin{array}{c}\text { Redes. Revista de Estudios para el Desarrollo de la } \\
\text { Comunicación }\end{array}$ & 3 & 4 & $(-)$ \\
\hline & $\mathrm{Br}$ & $292(190)$ & Rumores-Revista de Comunicação, Linguagem e Mídias & 3 & 4 & 2 \\
\hline & $\mathrm{Br}$ & $307(-)$ & Ciberlegenda & 3 & 3 & $(-)$ \\
\hline & Po & $307(-)$ & Comunicação e Sociedade & 3 & 3 & $(-)$ \\
\hline & Es & $307(-)$ & Historia y Comunicación Social & 3 & 3 & $(-)$ \\
\hline & $\mathrm{Br}$ & $307(192)$ & Linguagens-Revista de Letras, Artes e Comunicação & 3 & 3 & 2 \\
\hline & $\mathrm{Br}$ & $307(-)$ & Revista Internacional de Folkcomunicação & 3 & 3 & $(-)$ \\
\hline & Es & $307(185)$ & Signa & 3 & 3 & 3 \\
\hline & $\mathrm{Br}$ & $320(-)$ & Mediação (Online) Revista electrónica & 2 & 4 & $(-)$ \\
\hline & Es & $321(189)$ & Archivos de la Filmoteca & 2 & 3 & 3 \\
\hline & Es & $321(198)$ & Atalante & 2 & 3 & 2 \\
\hline & Es & $321(-)$ & Comunicació. Revista de Recerca i d'Anàlisi & 2 & 3 & $(-)$ \\
\hline & Per & $321(210)$ & Revista de Comunicación & 2 & 3 & 1 \\
\hline & $\mathrm{Br}$ & $329(-)$ & $\begin{array}{l}\text { Anagrama: Revista Científica Interdisciplinar da } \\
\text { Graduação }\end{array}$ & 2 & 2 & $(-)$ \\
\hline & $\mathrm{Br}$ & $329(198)$ & Comunicação \& Inovação & 2 & 2 & 2 \\
\hline & Bol & $329(-)$ & Punto Cero & 2 & 2 & $(-)$ \\
\hline
\end{tabular}




\begin{tabular}{|c|c|c|c|c|c|c|}
\hline \multirow{2}{*}{ C } & País & $\begin{array}{c}\text { Puesto } \\
\text { actual } \\
\text { (antes) }\end{array}$ & Revista & $\begin{array}{c}\text { h.5 } \\
\mathbf{2 0 0 8 /} \\
\mathbf{2 0 1 2}\end{array}$ & $\begin{array}{c}\text { Med. } \\
\text { ih.5 }\end{array}$ & $\begin{array}{c}\text { h.5 } \\
\mathbf{2 0 0 7 /} \\
\mathbf{2 0 1 1}\end{array}$ \\
\hline \multirow{6}{*}{4} & Es & $329(198)$ & Secuencias: Revista de historia del cine & 2 & 2 & 2 \\
\cline { 2 - 7 } & Ecu & $338(216)$ & Chasqui-Revista Latinoamericana de Comunicación & 1 & 2 & 1 \\
\cline { 2 - 7 } & Col & $338(212)$ & Revista Nexus Comunicación & 1 & 2 & 1 \\
\cline { 2 - 7 } & Chi & $345(-)$ & F@ro & 1 & 1 & $(-)$ \\
\cline { 2 - 7 } & Es & $345(216)$ & Making of: Cuadernos de Cine y Educación & 1 & 1 & 1 \\
\cline { 2 - 7 } & Es & $-(216)$ & Frame & $(-)$ & $(-)$ & 1 \\
\cline { 2 - 7 } & Es & $-(216)$ & Versión Original: Revista de cine & & & 1 \\
\cline { 2 - 7 } & & & & & \\
\hline
\end{tabular}

Fuente: Repiso y Delgado, 2013; Delgado y Cabezas-Clavijo, 2012. Elaboración propia.

\section{Análisis del Ih de las revistas de comunicación en Latinoamérica}

Brasil es la principal referencia en el ámbito Iberoamericano, además de España. Lidera el ranking con la revista con mayor índice $h$ de todas las de Iberoamérica, que en el anterior listado era la única en primer cuartil, dominado por Estados Unidos, el Reino Unido y Canadá. Su impacto se concentra en los cuartiles 2 y 3 .

Dos de cada tres de las revistas latinoamericanas tienen un impacto igual o inferior a cinco, lo que significa una media de una cita por año. Si exceptuamos las brasileñas, sólo cuatro revistas latinas superan ese nivel de impacto: dos colombianas (Palabra Clave y Signo y Pensamiento) y dos mexicanas (Razón y Palabra y Comunicación y Sociedad).

Las propuestas de EC3 se comparan con la evaluación de revistas que realiza el Ministerio de Educación de Brasil a través de, como se indicó, Qualis, un conjunto de procedimientos que desarrolla la Fundación Capes. Cada área de conocimiento o de evaluación clasifica las revistas científicas (la mayoría de las brasileñas vinculadas a programas de doctorado) con los indicadores A1 (máximo), A2, B1, B2, B3, B4, B5 y C (peso cero). Los resultados comparativos se exponen en la tabla 10.

En las revistas latinoamericanas que se publican en español, hay una clara estructura de impacto piramidal, con la mayoría de títulos concentra- 
Tabla 10

\section{Índice h5 de las revistas latinoamericanas de comunicación que se publican en portugués y su posición en el ranking internacional de revistas de comunicación}

\begin{tabular}{|c|c|c|c|c|c|c|}
\hline \multirow[b]{2}{*}{ País } & \multirow[b]{2}{*}{ Revista } & \multicolumn{3}{|c|}{ EC3. España } & \multicolumn{2}{|c|}{ Qualis. Brasil } \\
\hline & & $\begin{array}{c}h .5 \\
2008 / \\
2012\end{array}$ & $\begin{array}{r}\text { Med. } \\
\text { ih.5 } \\
\text { h.5 }\end{array}$ & $\begin{array}{c}\text { h.5 } \\
2007 / \\
2011\end{array}$ & $\begin{array}{c}\text { Áreas en } \\
\text { que se } \\
\text { evalúa }\end{array}$ & $\begin{array}{c}\text { Ciencias } \\
\text { sociales } \\
\text { aplicadas }\end{array}$ \\
\hline $\mathrm{Br}$ & Interface: Communication, Health, Education & 21 & 26 & 19 & 13 & No \\
\hline $\mathrm{Br}$ & MATRIZes & 9 & 14 & $(-)$ & 8 & $\mathrm{~A} 2$ \\
\hline $\mathrm{Br}$ & Revista FAMECOS: mídia, cultura e tecnologia & 9 & 14 & $(-)$ & 3 & A2 \\
\hline Po & Observatorio & 8 & 10 & $(-)$ & 5 & B2 \\
\hline $\mathrm{Br}$ & Comunicação Mídia e Consumo & 8 & 9 & 9 & 15 & B1 \\
\hline $\mathrm{Br}$ & Comunicação \& Educação & 7 & 11 & 8 & No & No \\
\hline $\mathrm{Br}$ & Intercom-Revista Brasileira de Ciências da Comunicação & 7 & 11 & 6 & 6 & $\mathrm{~A} 2$ \\
\hline $\mathrm{Br}$ & Comunicação em ciências da saúde & 7 & 10 & 8 & 11 & No aplica \\
\hline $\mathrm{Br}$ & Informação \& Sociedade & 7 & 8 & $(-)$ & 21 & Al \\
\hline $\mathrm{Br}$ & Líbero & 6 & 9 & $(-)$ & 7 & B1 \\
\hline $\mathrm{Br}$ & Estudos em Jornalismo e Mídia & 5 & 9 & 5 & 11 & B1 \\
\hline $\mathrm{Br}$ & Revista Organicom & 5 & 9 & $(-)$ & 5 & B1 \\
\hline $\mathrm{Br}$ & Brazilian Journalism Research & 5 & 8 & 5 & 11 & B1 \\
\hline $\mathrm{Br}$ & Eptic & 5 & 8 & $(-)$ & 20 & B1 \\
\hline $\mathrm{Br}$ & Comunicação \& Sociedade & 5 & 6 & 4 & 13 & B1 \\
\hline $\mathrm{Br}$ & Contemporanea-Revista de Comunicação e Cultura & 5 & 6 & 4 & 10 & B1 \\
\hline $\mathrm{Br}$ & Intexto Issn remite a Interface (Botucatu. Online) & 4 & 6 & $(-)$ & 13 & No aplica \\
\hline $\mathrm{Br}$ & Revista Contracampo & 4 & 6 & $(-)$ & 7 & B1 \\
\hline $\mathrm{Br}$ & Conexão & 4 & 5 & 4 & 4 & B1 \\
\hline $\mathrm{Br}$ & Distúrbios da Comunicação (2 Isbn) & 4 & 5 & 5 & 17 & \\
\hline & No aplica & & & & & \\
\hline $\mathrm{Br}$ & Em Questão & 4 & 5 & $(-)$ & 13 & B1 \\
\hline Po & DOC On-line. Revista digital de Cinema Documentario & 3 & 6 & 3 & 5 & B1 \\
\hline $\mathrm{Br}$ & Contemporânea5 & 3 & 5 & - & 4 & B1 \\
\hline $\mathrm{Br}$ & Discursos Fotográficos & 3 & 5 & $(-)$ & $\begin{array}{c}10 \\
3 \text { online }\end{array}$ & $\begin{array}{l}\text { B1 } \\
\text { B1 }\end{array}$ \\
\hline $\mathrm{Br}$ & Rumores-Revista de Comunicação, Linguagem e Mídias & 3 & 4 & 2 & 9 & B1 \\
\hline $\mathrm{Br}$ & Linguagens-Revista de Letras, Artes e Comunicaçao & 3 & 3 & 2 & 6 & B5 \\
\hline $\mathrm{Br}$ & Ciberlegenda & 3 & 3 & $(-)$ & 7 & $\mathrm{~B} 1$ \\
\hline Po & Comunicação e Sociedade & 3 & 3 & $(-)$ & - & No aplica \\
\hline $\mathrm{Br}$ & Revista Internacional de Folkcomunicação & 3 & 3 & $(-)$ & 7 & B3 \\
\hline
\end{tabular}




\begin{tabular}{|c|c|c|c|c|c|c|}
\hline \multirow[b]{2}{*}{ País } & \multirow[b]{2}{*}{ Revista } & \multicolumn{3}{|c|}{ EC3. España } & \multicolumn{2}{|c|}{ Qualis. Brasil } \\
\hline & & $\begin{array}{c}h .5 \\
2008 / \\
2012\end{array}$ & $\begin{array}{r}\text { Med. } \\
\text { ih.5 } \\
h .5\end{array}$ & $\begin{array}{c}h .5 \\
2007 / \\
2011\end{array}$ & $\begin{array}{l}\text { Áreas en } \\
\text { que se } \\
\text { evalúa }\end{array}$ & $\begin{array}{l}\text { Ciencias } \\
\text { sociales } \\
\text { aplicadas }\end{array}$ \\
\hline $\mathrm{Br}$ & $\begin{array}{c}\text { Mediação (Online) Revista eletrônica } \\
\text { Belo Horizonte } \\
\text { Curitibia } \\
\text { Pires do Rio }\end{array}$ & 2 & 4 & $(-)$ & $\begin{array}{l}6 \\
6 \\
6\end{array}$ & $\begin{array}{c}\text { B3 } \\
\text { B5 } \\
\text { No aplica }\end{array}$ \\
\hline $\mathrm{Br}$ & Comunicação \& Inovação & 2 & 2 & 2 & 7 & B1 \\
\hline $\mathrm{Br}$ & Anagrama & 2 & 2 & $(-)$ & 7 & B5 \\
\hline
\end{tabular}

Fuente: Qualis (CAPES); Repiso y Delgado (2013); Delgado y Cabezas-Clavijo, 2012. Elaboración propia.

dos en los cuartiles 3 y 4. Existe la presencia de revistas de comunicación de Colombia, México, Chile, Venezuela, Perú, Bolivia y Ecuador. Nos sorprende la ausencia de revistas publicadas en Argentina; así como de Uruguay, de Paraguay y de todos los países de Centroamérica.

La revisión del listado en octubre de 2013 incorpora publicaciones de Colombia, Chile, Bolivia, México y Venezuela. También hay nuevos títulos de revistas de España, Brasil y Portugal. Desaparecen Frame y Versión Original (España). Las dos estaban en el cuarto cuartil, con ih5=1. También falta en el listado de 2013 Economia Política das Tecnologias da Informação e da Comunicação, de Brasil (que estaba en el tercer cuartil con ih5 de 6), y lo que más sorprendente es que no está Perspectivas em Ciência da Informação, que en 2012 estaba de primera en el segundo cuartil, con ih5 de 11.

\section{Conclusiones}

El índice $h$ es un indicador bibliométrico que gana fuerza como referente de la trayectoria investigadora en comparación con el factor de impacto principalmente porque aúna difusión e impacto. Su principal debilidad es que no aprecia las colas de citas por encima ni por debajo del índice y promociona a los productores masivos de artículos en tanto que penaliza a los selectivos.

Las bases de datos de rastreo condicionan el resultado de índice $h$ de un autor o revista. ISI, SCOPUS y GSM dan a un mismo investigador índices $h$ diferentes y reconocen citas distintas para un mismo artículo. GSM 
Tabla 11

\section{Índice h5 de las revistas latinoamericanas de comunicación que se publican en español y su posición en el ranking internacional de revistas de comunicación}

\begin{tabular}{|c|c|c|c|c|c|c|}
\hline $\mathrm{C}$ & País & $\begin{array}{l}\text { Puesto } \\
\text { actual } \\
\text { (antes) }\end{array}$ & Revista & $\begin{array}{c}h .5 \\
2008 / \\
2012\end{array}$ & $\begin{array}{r}\text { Med. } \\
\text { ih.5 }\end{array}$ & $\begin{array}{c}\text { h.5 } \\
2007 / \\
2011\end{array}$ \\
\hline 1 & - & - & - & - & - & - \\
\hline \multirow{2}{*}{2} & Mex & $157(-)$ & Razón y Palabra & 7 & 9 & $(-)$ \\
\hline & Col & $175(-)$ & Palabra Clave & 7 & 7 & $(-)$ \\
\hline \multirow{6}{*}{3} & Col & $184(-)$ & Signo y Pensamiento & 6 & 9 & $(-)$ \\
\hline & Mex & $193(131)$ & Comunicación y Sociedad & 6 & 7 & 6 \\
\hline & Ven & $241(-)$ & Opción & 4 & 9 & $(-)$ \\
\hline & Per & $241(143)$ & Diálogos de la Comunicación & 4 & 9 & 5 \\
\hline & Ven & $244(-)$ & REDHECS & 4 & 8 & $(-)$ \\
\hline & Chi & $251(-)$ & Cuadernos de Información & 4 & 6 & $(-)$ \\
\hline \multirow{8}{*}{4} & $\mathrm{Es} / \mathrm{Ve}$ & $281(192)$ & $\begin{array}{l}\text { Anuario Electrónico de Estudios en Comunicación } \\
\text { Social. "Disertaciones" }\end{array}$ & 3 & 6 & 2 \\
\hline & Mex & $285(-)$ & Cineforum & 3 & 5 & $(-)$ \\
\hline & Ven & $292(182)$ & Comunicación: Estudios Venezolanos de Comunicación & 3 & 4 & 3 \\
\hline & Per & $321(210)$ & Revista de Comunicación & 2 & 3 & 1 \\
\hline & Bol & $329(-)$ & Punto Cero & 2 & 2 & $(-)$ \\
\hline & $\mathrm{Ecu}$ & $338(216)$ & Chasqui-Revista Latinoamericana de Comunicación & 1 & 2 & 1 \\
\hline & Col & $338(212)$ & Revista Nexus Comunicación & 1 & 2 & 1 \\
\hline & Chi & $345(-)$ & F@ro & 1 & 1 & \\
\hline
\end{tabular}

Fuente: Repiso y Delgado (2013); Delgado y Cabezas-Clavijo, 2012. Elaboración propia.

está universalizando el acceso gratuito a los resultados bibliométricos de difusión de la investigación pero aún ofrece resultados no plenamente fiables principalmente por la opacidad en las fuentes que rastrea y por los criterios de clasificación de áreas y revistas.

Los perfiles de actividad investigadora en GSM han logrado una amplia penetración en un corto periodo de tiempo por ser de acceso abierto y de sencillo manejo para compilar y visibilizar trayectorias individuales o grupales exportables y referenciables con url. 
El área de comunicación en España ofrece bajos índices $h$ de revistas e investigadores. La mediana $h$ de las revistas de comunicación españolas en 2012 es 4. No admite comparación entre áreas pero, a modo ilustrativo, el índice $h$ más alto entre las revistas científicas en español triplica el índice de la revista de comunicación mejor situada. Al hallar el $h$ del área de comunicación en España, el resultado es 17 (sobre un mínimo de 16), mientras en áreas afines, como economía, estaría en 50. La revista española de comunicación con mayor índice reconocido por GSM tiene un indicador $h$ de 13.

Estos datos no sólo evidencian la falta de plataformas de difusión científica sino que delatan un déficit en la gestión de políticas de difusión de la investigación, en la que deben implicarse individualmente cada investigador y colectivamente los grupos y equipos e instituciones en las que se integran. El auge del índice $h$ parece abrir una nueva etapa en la labor de los equipos de investigación que no concluye en la difusión, ya que incluye la gestión de la comunicación sobre la investigación realizada.

\section{Referencias}

Alonso, S., Cabrerizo F. J., Herrera-Viedma, E. y Herrera, F. (2009). "h-Index: A review focused in its variants, computation and standardization for different scientific fields". En: Journal of Informetrics, 3, pp. 273-89. Disponible en: http://sci2s.ugr.es/hindex/pdf/JOI3-4-273-289.pdf [fecha de consulta: 22 de diciembre de 2013].

Clavijo, A., Jiménez-Contreras, E. y Ruiz-Pérez, R. (2012). “El índice h de las revistas españolas de Ciencias Sociales según Google Scholar (2001- 2010)”. En: EC3 Informes, abril 2012.

De Pablos Coello, J. M., Mateos Martín, C. y Túñez López, M. (2013). "Google, cambia el paradigma de la métrica científica". En: Historia y Comunicación Social, 18, pp. 225-235.

Delgado López-Cozar, E., Orduña-Malea, E., Marcos Cartagena, D., Jiménez Contreras, E. y Ruiz Pérez, R. (2012). "JOURNAL SCHOLAR: Una alternativa internacional, gratuita y de libre acceso para 
medir el impacto de las revistas de Arte, Humanidades y Ciencias Sociales”. En: EC3 Working Papers, 5. Disponible en: http://digibug.ugr.es/handle/10481/20375 [fecha de consulta: 18 de diciembre de 2013].

Delgado López-Cozar, E. (2012). “De abril a noviembre: el crecimiento de Google Scholar Metrics”. En: EC3 Working Papers, 9. Disponible en: http://digibug.ugr.es/bitstream/10481/22466/1/Google Scholar_Metrics.pdf[fecha de consulta: 17 de diciembre de 2013].

Delgado López-Cozar, E. y Cabezas-Clavijo, A. (2012). "Google Scholar Metrics revisado: Ahora empieza a ir en serio”. En: EC3 Working Papers, 8.

Delgado López-Cozar, E. y Repiso Caballero, R. (2012). “Índice H de las revistas de Comunicación según Google Scholar Metrics (20072011)”. En: EC3 Miscelánea. Disponible en: http://digibug.ugr.es/ handle/10481/22483 [fecha de consulta: 19 de diciembre de 2013].

Delgado López-Cozar, E., Robinson-García, N. y Torres-Salinas, D. (2012). "Manipular Google Scholar Citations y Google Scholar Metrics: simple, sencillo y tentador”. En: EC3 Working Papers. Disponible en: http://digibug.ugr.es/bitstream/10481/20469/1/scholar.pdf [fecha de consulta: 20 de diciembre de 2013].

Delgado López-Cózar, E. y Torres Salinas, D. (2013). “Cómo utilizar Google Scholar para mejorar la visibilidad de tu producción científica”. Grupo EC3, Granada. Disponible en: http://digibug.ugr.es/ handle/10481/23794 [fecha de consulta: 3 de diciembre de 2013].

Delgado López-Cózar, E. y Repiso Caballero, R. (2012). “Índice H de las revistas de Comunicación según Google Scholar Metrics (20072011)”. En: EC3 Miscelánea.

Delgado López-Cózar, E. y Repiso Caballero, R. (2013). "El impacto de las revistas de comunicación: comparando Google Scholar Metrics, 
Web of Science y Scopus”. En: Comunicar, 41 (XXI), pp. 45-52. Disponible en: http://dx.doi.org/10.3916/C41-2013-04 [fecha de consulta: 22 de noviembre de 2013].

Delgado López-Cozar, E., Orduña-Malea, E., Marcos Cartagena, D., Jiménez-Contreras, E. y Ruiz-Pérez, R. (2007). "El factor de impacto de las revistas científicas: limitaciones e indicadores alternativos". En: El profesional de la información, 16 (1), pp. 4-11.

Dorta-Gonzalez, P. y Dorta-González, M. I. (2010). "Indicador bibliométrico basado en el índice h". En: Revista Española de Documentación Cientifica, 33, pp. 225-245.

Egghe, L. (2006). “Theory and practise of the g-index”. En: Scientometrics, 69 (1), pp. 131-162.

Garfield, E. (2006). "The history and meaning of the journal impact factor”. En: Jama, 295 (1), pp. 90-93.

Hirsch, J. E. (2005). "An index to quantify an individual's scientific output". En: Proceedings of the National Academy of Sciences of the United States of America, 102, pp. 16569-16572.

Hirsch, J. E. (2012): “An index to quantify an individual's scientific research output that takes into account the effect of multiple coauthorship". En: Scientometrics, 85, pp. 741-754.

Imperial,J.y Rodríguez-Navarro, A. (2005). "Utilidad del índice h de Hirsch para evaluar la investigación en España”. Disponible en: http:// www.bit.etsia.upm.es/Imperial_Rodriguez-Navarro.pdf [fecha de consulta: 14 de noviembre de 2013].

Imperial, J. y Rodríguez-Navarro, A. (2007). “Usefulness of hirsch’s h-index to evaluate scientific research in Spain”. En: Scientometrics, 71 (2), pp. 271-282. 
Roncallo-Dow, S., Uribe-Jongbloed, E. y Calderón-Reyes, I. (2013). “La investigación en comunicación: Los límites y limitantes del conocimiento". En: Co-herencia, 10 (18), pp. 161-187.

Túñez López, M. (2013). “El 'índice h’ de la investigación en Comunicación en España, Portugal y Latinoamérica: Web of Knowledge (Wok), Scopus y Google Scholar”. En: Communication \& Society/Comunicación y Sociedad, 26 (4), pp. 53-75.

Túñez, M. y De Pablos, J. M. (2013). “El 'índice h’ en las estrategias de visibilidad, posicionamiento y medición de impacto de artículos y revistas de investigación”. En: Actas del II Congreso Nacional sobre Metodología de la Investigación en Comunicación y del Simposio Internacional sobre Política Científica en Comunicación (pp. 133150). Universidad de Valladolid: Segovia. Disponible en: http:// uvadoc.uva.es/handle/10324/2996 [fecha de consulta: 3 de noviembre de 2013]. 